\title{
Single bouts of exercise selectively sustain attentional processes
}

\author{
MATTHEW B. PONTIFEX, ${ }^{a}$ ANDREW C. PARKS, ${ }^{a}$ DAVID A. HENNING,,${ }^{a}$ AND KEITA KAMIJO ${ }^{b}$ \\ aDepartment of Kinesiology, Michigan State University, East Lansing, Michigan, USA \\ ${ }^{\mathrm{b} S} \mathrm{School}$ of Sport Sciences, Waseda University, Tokyo, Japan
}

\begin{abstract}
This study examined how single bouts of exercise may differentially modulate neuroelectric correlates of attentional orienting and processing. Using a within-participants design, ERPs and task performance were assessed in response to a perceptually challenging three-stimulus oddball task prior to and following a bout of exercise or seated rest during two separate, counterbalanced sessions. Findings revealed that, following a single bout of exercise, attentional processing was sustained relative to pretest whereas prolonged sitting resulted in attentional decrements. Focal attention resulting from attentional orienting, in contrast, does not appear to be sensitive to the influences of single bouts of physical activity. These findings suggest that acute exercise-induced changes in cognition do not originate from an overall modulation of attention but instead are specific to aspects of attentional processing.
\end{abstract}

Descriptors: Acute exercise, Stimulus discrimination, Attentional orienting, P300, P3a, P3b

The effect of single bouts of exercise on cognition has been increasingly assessed over the past decade, with evidence observing enhanced cognitive performance following an acute bout of exercise (Lambourne \& Tomporowski, 2010; Verburgh, Königs, Scherder, \& Oosterlaan, 2014). Initial articulation of the neural mechanisms underlying these exercise-induced changes in cognition has been obtained using ERP. Such methods provide a means of gaining insight into the effects of single bouts of physical activity on cognition through the examination of a subset of processes that occur between stimulus encoding and response production. In particular, the P300-a large positive-going peak occurring approximately 300 to $800 \mathrm{~ms}$ following stimulus onset-has garnered considerable attention in the literature given that it provides an index of attentional processes (Polich, 2007). Although the P300 is comprised of two distinct subcomponents, the P3a and the P $3 b$, to date, research in this area has focused exclusively on the P3b ERP component. Given neuropharmacological evidence suggesting that these components may relate to different neurotransmitter systems (Nieuwenhuis, Aston-Jones, \& Cohen, 2005), the investigation of how these single bouts of physical activity may differentially modulate the P3a and P3b ERP components may provide further insight into the mechanisms of exercise-induced changes in cognition.

The P3a and P3b ERP components are further distinguished by the context in which a stimulus occurs, which dictates their char-

Support for the preparation of this manuscript was provided by a grant from the Eunice Kennedy Shriver National Institute of Child Health and Human Development (NICHD) to MBP (R21 HD078566) and a Summer Research Development Fellowship awarded to ACP through the College of Education and the Graduate School at Michigan State University.

Address correspondence to: Matthew B. Pontifex, Ph.D., Department of Kinesiology, 27P IM Sports Circle, Michigan State University, East Lansing, MI 48824-1049, USA. E-mail: pontifex@msu.edu acteristic amplitude and scalp topography. For example, one such task, which has been found to reliably elicit both P300 components, is the three-stimulus oddball task. In this task, participants are instructed to respond to (or count) an infrequent target stimulus while ignoring either a frequently presented stimulus or an infrequent distractor stimulus. The P3a ERP component is an endogenous component elicited in the absence of task instructions when the infrequent distractor stimulus occurs. The P3a is therefore thought to be associated with the selection of stimulus information governed by attentional orienting (Knight, 1984; Kok, 2001; Rushby, Barry, \& Doherty, 2005), reflecting the disengagement of previous attentional focus and subsequent reengagement of attentional processes towards the infrequent stimulus (Squires, Squires, \& Hillyard, 1975). Thus, the amplitude of the P3a provides an index of attentional orienting with increased amplitude related to greater engagement of focal attention (Polich, 2007). In contrast, the $\mathrm{P} 3 \mathrm{~b}$ is elicited when a participant is instructed to respond to (or count) the infrequently presented target stimulus. This ERP component is thought to reflect neuronal activity associated with the revision of the mental representation of the previous event (Donchin, 1981), such that the P3b is sensitive to the allocation of attentional resources during stimulus engagement (Polich, 2007). Accordingly, based on a theoretical account of the P3 by Polich (2007), the amplitude of the P3b is believed to be proportional to the resources allocated towards the suppression of extraneous neuronal activity in order to facilitate attentional processing. P3b timing — marked by the latency of the peak amplitude — is generally considered as a measure of stimulus detection and classification speed (Ilan \& Polich, 1999; Magliero, Bashore, Coles, \& Donchin, 1984).

The P3b has been shown to be a useful tool in characterizing the effects of single bouts of physical activity on cognition, particularly in instances where the cognitive task may not sufficiently tax neural resources to engender behavioral differences (Hillman, Kamijo, \& 
Pontifex, 2012). Indeed, exercise-induced modulations of both the amplitude and latency of the P3b ERP component have been observed across multiple tasks and participant populations (Hillman, Kamijo, \& Pontifex, 2012). Within the relevant research investigating the effect of single bouts of physical activity on stimulus discrimination using oddball tasks, a generally beneficial influence of aerobic modalities of exercise is evident such that moderate intensity aerobic exercise appears to relate to larger P3b amplitude (Kamijo et al., 2004; Nakamura, Nishimoto, Akamatu, Takahashi, \& Maruyama, 1999) and shorter P3b latency (Kumar, Singh, Sakshi, Roy, \& Behera, 2012). Such findings suggest that a single bout of aerobic exercise may promote an increased ability to inhibit neuronal activity unrelated to task performance, which would facilitate stimulus evaluation resulting in increases in the amplitude and reductions in latency for the P3b ERP component.

However, a critical limitation of the existent literature in this area is the reference point utilized to determine exercise effects on cognition (Lambourne \& Tomporowski, 2010), with research designs utilizing either a within-subjects postexperimental condition comparison (Hillman, Snook, \& Jerome, 2003; Hillman et al., 2009; Kamijo et al., 2004, 2009; Pontifex, Saliba, Raine, Picchietti, \& Hillman, 2013; Scudder, Drollette, Pontifex, \& Hillman, 2012) or a between-subjects pre-post exercise comparison (Kumar et al., 2012; Magnié et al., 2000; Nakamura et al., 1999; Yagi, Coburn, Estes, \& Arruda, 1999). In the former, while every subject engages in both experimental conditions, problematically, day-to-day variations in biological and environmental determinants to the generation of the P3b may confound the interpretation of observed findings after exercise relative to after rest (Polich \& Kok, 1995); whereas in the latter, such a design fails to account for any modulations in the $\mathrm{P} 3 \mathrm{~b}$, which may occur simply as a product of repeated exposure unrelated to the exercise stimulus. Although it is important to note that the specific aspect of cognition assessed differs across these various investigations, methodologically, the lack of suitable pre-post comparisons for both exercise and control conditions applies regardless of the type of cognition assessed. Therefore, in order to determine how single bouts of physical activity may differentially influence specific aspects of attention, a second aim of the present study was to ameliorate such methodological limitations. Toward this end, a within-subjects repeated measures design was utilized to examine neuroelectric indices of attention prior to and immediately following moderate intensity aerobic exercise and a resting control, employing a well-studied stimulus discrimination task to elicit reliable $\mathrm{P} 3 \mathrm{a}$ and $\mathrm{P} 3 \mathrm{~b}$ components (Conroy \& Polich, 2007; Polich, 2007; Walhovd, Rosquist, \& Fjell, 2008). This approach permitted the direct evaluation of theoretically well-developed techniques to determine the effect of a single bout of physical activity on attentional processes. It was hypothesized that, consonant with the existent literature, physical activity would be associated with facilitation in attentional processes such that a bout of exercise would result in a greater increase in the amplitude, and decrease in the latency, of both the P3a and P3b components relative to changes observed following the bout of seated rest.

\section{Method}

\section{Participants}

Thirty-four college-aged young adults (18 female; $19.3 \pm 0.9$ years) participated in this investigation at Michigan State Univer- sity. An original sample of 39 participants was recruited, with five participants failing to complete all testing sessions (two female). Only participants who completed all sessions were included in statistical analysis. All participants provided written informed consent in accordance with the Institutional Review Board at Michigan State University and reported being free of any neurological disorder, psychological condition, previous history of head trauma, cardiovascular disease, physical disabilities, and indicated normal or corrected-to-normal vision.

\section{Task}

A perceptually challenging three-stimulus oddball task was used in which participants were instructed to respond as quickly and accurately as possible with a right-hand thumb press only to an infrequent target stimulus while ignoring all other stimuli (Hagen, Gatherwright, Lopez, \& Polich, 2006; Polich \& Criado, 2006; Pontifex, Hillman, \& Polich, 2009). Target stimuli were 55-mm diameter white circles occurring with a probability of 0.12 , while nontarget stimuli were $47-\mathrm{mm}$ diameter white circles presented with a probability of 0.76 . Additionally, a full-screen checkerboard was used as a distractor stimulus presented with a probability of 0.12 . All stimuli were presented focally on a computer monitor at a distance of $1 \mathrm{~m}$ for $100 \mathrm{~ms}$, with a 1,000-ms response window and a 1,700-ms intertrial interval using PsychoPy, 1.76 (Peirce, 2009). Three blocks of 175 trials were presented, resulting in a total of 63 target and distractor stimuli.

\section{ERP Recording}

EEG activity was recorded from 64 electrode sites arranged in an extended montage based on the International 10-10 system (Chatrian, Lettich, \& Nelson, 1985) using a Neuroscan Quik-Cap (Compumedics, Inc., Charlotte, NC). Recordings were referenced to averaged mastoids (M1, M2), with AFz serving as the ground electrode and impedance less than $10 \mathrm{k} \Omega$. In addition, electrodes were placed above and below the left orbit and on the outer canthus of both eyes to monitor electrooculographic (EOG) activity with a bipolar recording. Continuous data were digitized at a sampling rate of $1000 \mathrm{~Hz}$ and amplified 500 times with a DC to $70 \mathrm{~Hz}$ filter using a Neuroscan SynAmps RT amplifier. Continuous data were corrected offline for EOG artifacts using a spatial filter, which performed a spatial singular value decomposition based on principal component analysis to determine the major components that characterized the EOG artifact across all channels. Channels were then reconstructed without the artifact component (Compumedics Neuroscan, 2003). Stimulus-locked epochs were created for correct trials from -100 to $1,000 \mathrm{~ms}$ around the stimulus, baseline corrected using the prestimulus period, and filtered using a zero phase shift low-pass filter at $30 \mathrm{~Hz}(24 \mathrm{~dB} /$ octave). Artifact in the EEG signal was identified if an amplitude excursion of $\pm 75 \mu \mathrm{V}$ occurred or if the overall variance of an epoch exceeded more than 1.5 standard deviations of the mean variance of all accepted epochs. Artifact-free trials that were accompanied by correct responses were averaged. The P3 components were evaluated as the mean amplitude within a 50-ms interval surrounding the largest positive-going peak within a $300-700 \mathrm{~ms}$ latency window (Pontifex et al., 2013). Amplitude was measured as the difference between the mean prestimulus baseline and mean peak-interval amplitude, while peak latency was defined as the time point corresponding to the maximum peak amplitude. 


\section{Procedure}

Using a within-participants design, participants visited the laboratory on 2 separate days (mean days apart, $5.2 \pm 5.1$ days; mean time of day difference, $1.2 \pm 1.4 \mathrm{~h}$ ). Following provision of informed consent, participants completed a health history/demographics questionnaire, the Physical Activity Readiness Questionnaire (Thomas, Reading, \& Shephard, 1992), and the Edinburgh Handedness Inventory (Oldfield, 1971). Participants were then counterbalanced into two different session orders (day 1: sitting, day 2: exercise or day 1: exercise, day 2: sitting) to ensure that any observed effects were unrelated to the specific order in which participants received the exercise and sitting conditions. The experimental conditions consisted of $20 \mathrm{~min}$ of either sitting $\left(\mathrm{HR}_{\text {during siting }}=70.4 \pm 10.0 \mathrm{bpm}\right)$ or exercise on a motor-driven treadmill at an aerobic exercise intensity of approximately $70 \%$ of age-predicted $(220$ - age $)$ maximum heart rate $\left(\mathrm{HR}_{\text {during exercise }}=\right.$ $138.8 \pm 11.0 \mathrm{bpm}$ ). A polar HR monitor (Model H7, Polar Electro, Finland) was used to measure HR throughout the test. To ensure that any observed effects were unrelated to experimenter interaction or nonexercise-related stimuli, during each experimental condition participants watched an emotionally neutral video (minutes 65-85 and 85-105 from Wonders of the Universe, Cooter, Holt, \& Lachmann, 2011). Participants were seated in a sound-attenuated testing chamber where ERPs and task performance were assessed in response to the perceptually challenging three-stimulus oddball task prior to each experimental condition $(18.0 \pm 4.9$ min prior to sitting; $19.4 \pm 5.7 \mathrm{~min}$ prior to exercise), and again once heart rate returned to within $10 \%$ of preexperimental condition levels ( $3.3 \pm 1.8 \mathrm{~min}$ postsitting; $4.6 \pm 3.0 \mathrm{~min}$ postexercise).

\section{Statistical Analysis}

All statistical analyses were conducted with $\alpha=.05$ using the Greenhouse-Geisser statistic with subsidiary univariate analyses of variance (ANOVAs) and Bonferroni-corrected $t$ tests for post hoc comparisons using PASW Statistics, 19.0 (IBM, Armonk, NY). Given a sample size of 34 participants and beta of .20 (i.e., $80 \%$ power), the present research design theoretically had sufficient sensitivity to detect multivariate repeated measures effects exceeding $f=0.248$ (assuming correlation between repeated measures $\geq 0.5$ ) and $t$-test differences exceeding $d=0.495$ (with a two-sided alpha) as computed using G*Power 3.1.2 (Faul, Erdfelder, Lang, \& Buchner, 2007). Analysis of task performance measures (reaction time [RT] and response accuracy to the target stimuli) were conducted separately using a 2 (Mode: sitting, exercise) $\times 2$ (Time: pretest, posttest) multivariate repeated measures ANOVA. The P3a and P3b ERP components were assessed separately for amplitude and latency using a 2 (Mode: sitting, exercise) $\times 2$ (Time: pretest, posttest) $\times 7$ (Site: Fz, FCz, Cz, CPz, $\mathrm{Pz}, \mathrm{POz}, \mathrm{Oz}$ ) multivariate repeated measures ANOVA (Pontifex et al., 2013). To ensure that any potential findings were not masked by differences in pretest performance, analyses were also conducted replicating the models listed above but collapsing time into a change score (posttest minus pretest; Hillman et al., 2014).

\section{Results}

Following examination of all data to ensure the assumption of normality was met, preliminary analyses were performed separately to test whether sex or session order related to any behavioral or neuroelectric variables by including them as an additional between-subjects factor within the multivariate repeated measures ANOVA models. Findings revealed no significant interactions with mode or time ( $p s \geq .07$ ); thus, all further analyses were collapsed across sex and session order. Preliminary analyses were also conducted to ensure that any findings were not the result of different numbers of trials included in the ERP averages. Analyses revealed no significant differences in the number of trials included for the P3b ERP component (sitting pretest: $37.5 \pm 6.6$; sitting posttest: $37.4 \pm 6.2$; exercise pretest: $39.5 \pm 6.9$; exercise posttest: $37.5 \pm 6.1 ; p \mathrm{~s} \geq .07$ ) or for the P3a ERP component (sitting pretest: $41.5 \pm 7.9$; sitting posttest: $39.7 \pm 6.7$; exercise pretest: $41.3 \pm 7.6$; exercise posttest: $39.6 \pm 7.6 ; p \mathrm{~s} \geq .06$ ).

\section{Behavioral Performance}

Table 1 provides mean $(S D)$ behavioral and neuroelectric measures as a function of mode and time. Analysis of RT revealed a main effect of mode, $F(1,33)=4.6, p=.04, \eta^{2}=.12$, with shorter RT for the exercise $(439.3 \pm 39.3 \mathrm{~ms})$ relative to the sitting $(449.9 \pm 44.7 \mathrm{~ms})$ condition. No main effects of time, $F(1,33)=0.6, p=.46, \eta^{2}=.02$, or interactions of Mode $\times$ Time, $F(1,33)=2.6, p=.12, \eta^{2}=.07$, were observed. No significant differences were observed between the change in RT (posttest RT minus pretest RT) for exercise relative to the change in RT for sitting, $t(33)=1.6, p=.12, d=0.9$. Analysis of response accuracy revealed a main effect of time, $F(1,33)=13.2$, $p=.001, \eta^{2}=.29$, with decreased response accuracy at posttest $(84.9 \pm 8.8 \%)$ relative to pretest $(88.1 \pm 6.6 \%)$. No main effects of mode, $F(1,33)=0.6, p=.43, \eta^{2}=.02$, or interactions of Mode $\times$ Time, $F(1,33)=0.3, p=.59, \eta^{2}=.01$, were observed. Further, no significant differences were observed between the change in response accuracy (posttest response accuracy minus pretest response accuracy) for exercise relative to the change in response accuracy for sitting, $t(33)=0.5, p=.59, d=0.08$.

Table 1. Mean (SD) Behavioral and Neuroelectric Characteristics for Mode and Time

\begin{tabular}{|c|c|c|c|c|}
\hline \multirow[b]{2}{*}{ Measure } & \multicolumn{2}{|c|}{ Sitting } & \multicolumn{2}{|c|}{ Exercise } \\
\hline & Pretest & Posttest & Pretest & Posttest \\
\hline Response accuracy (\% correct) & $87.7 \pm 9.4$ & $84.0 \pm 11.0$ & $88.5 \pm 8.3$ & $85.7 \pm 8.5$ \\
\hline P3b amplitude $(\mu \mathrm{V})$ & $12.3 \pm 5.8$ & $10.6 \pm 5.3$ & $11.9 \pm 6.9$ & $12.0 \pm 6.2$ \\
\hline P3b latency (ms) & $417.4 \pm 39.0$ & $422.2 \pm 41.9$ & $410.9 \pm 40.4$ & $421.2 \pm 49.3$ \\
\hline P3a amplitude $(\mu \mathrm{V})$ & $16.4 \pm 5.6$ & $13.8 \pm 4.0$ & $16.4 \pm 5.9$ & $14.8 \pm 4.8$ \\
\hline P3a latency (ms) & $350.6 \pm 20.6$ & $351.3 \pm 19.9$ & $352.2 \pm 20.9$ & $351.9 \pm 23.1$ \\
\hline
\end{tabular}

Note. Mean values reported for reaction time and response accuracy are in response to the target stimulus. Means values reported for P3b and P3a ERP components are collapsed across electrode site as site was not observed to interact with mode or time $(p s \geq .08)$. 


\section{P3b ERP Component}

Figure 1 illustrates the grand-average ERP waveform for each mode and time point. Analysis of $\mathrm{P} 3 \mathrm{~b}$ amplitude revealed a main effect of time, $F(1,33)=4.0, p=.05, \eta^{2}=.11$, which was super- seded by an interaction of Mode $\times$ Time, $F(1,33)=12.8, p=.001$, $\eta^{2}=.28$. Decomposition of the Mode $\times$ Time interaction revealed reductions in $\mathrm{P} 3 \mathrm{~b}$ amplitude between pre- and posttest in the sitting condition, $t(33)=3.2, p=.003, d=0.06$; whereas no changes in $\mathrm{P} 3 \mathrm{~b}$ amplitude were observed between pre- and posttest in the

\section{Target Stimulus}

$\mathrm{Fz}$
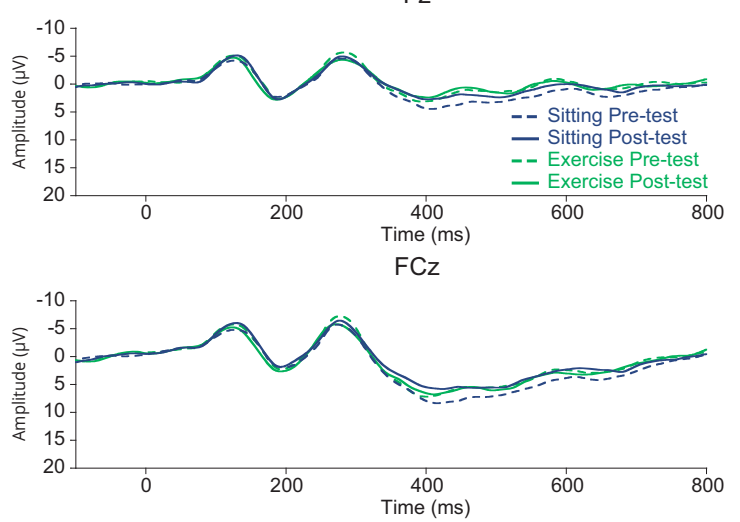

$\mathrm{Cz}$
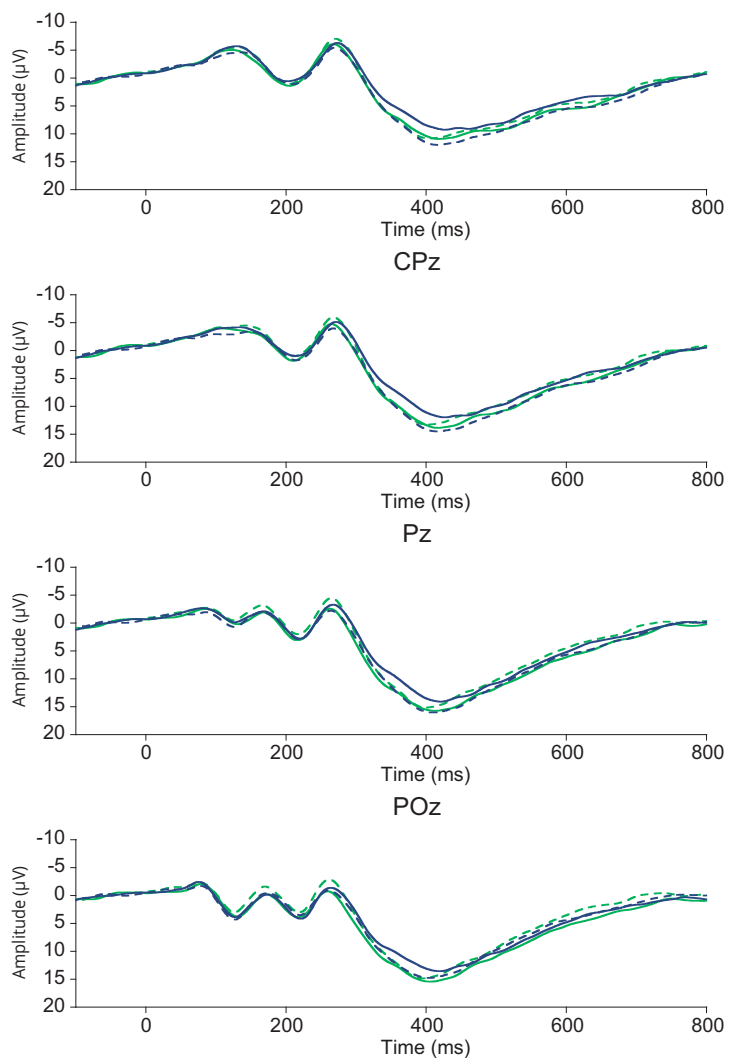

$\mathrm{Oz}$

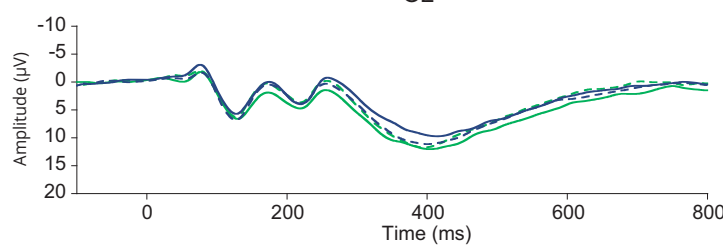

Distractor Stimulus

$\mathrm{Fz}$
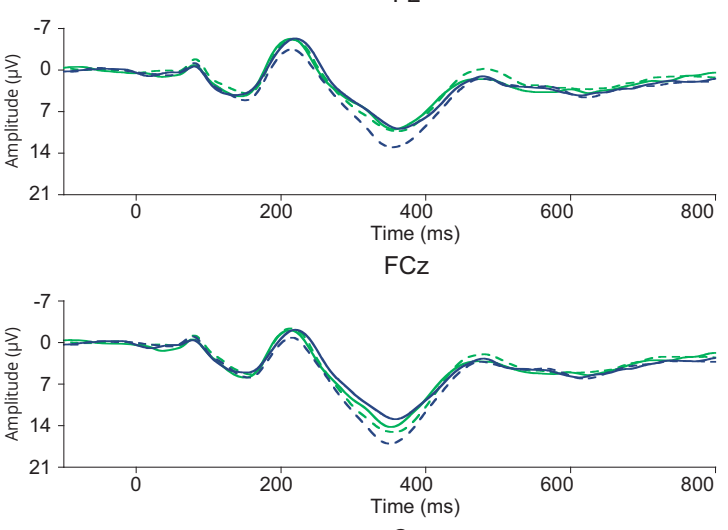

$\mathrm{Cz}$
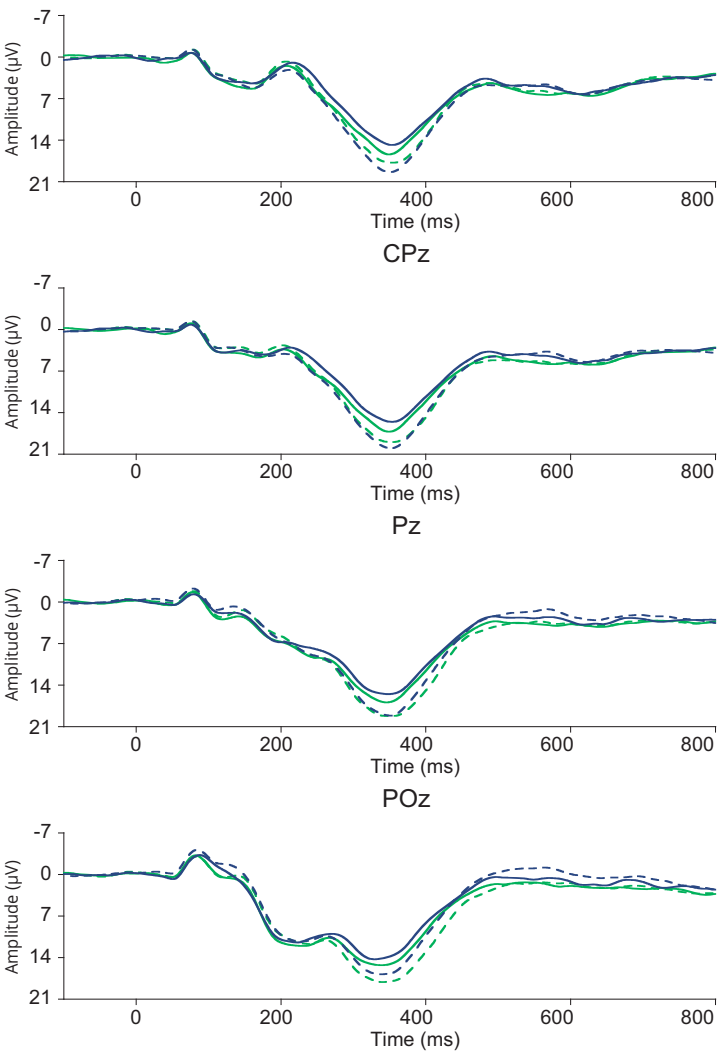

$\mathrm{Oz}$

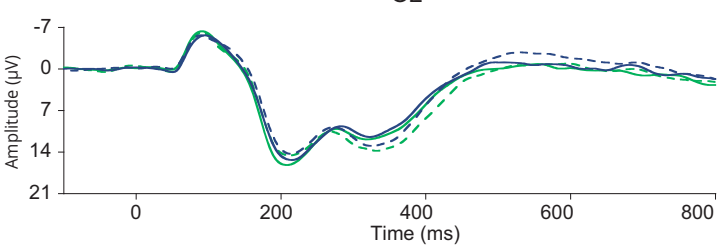

Figure 1. Grand-average ERP waveforms for each mode and time point to the target (i.e., P3b) and distracter (i.e., P3a) stimulus. Note different voltage scales for each component. 

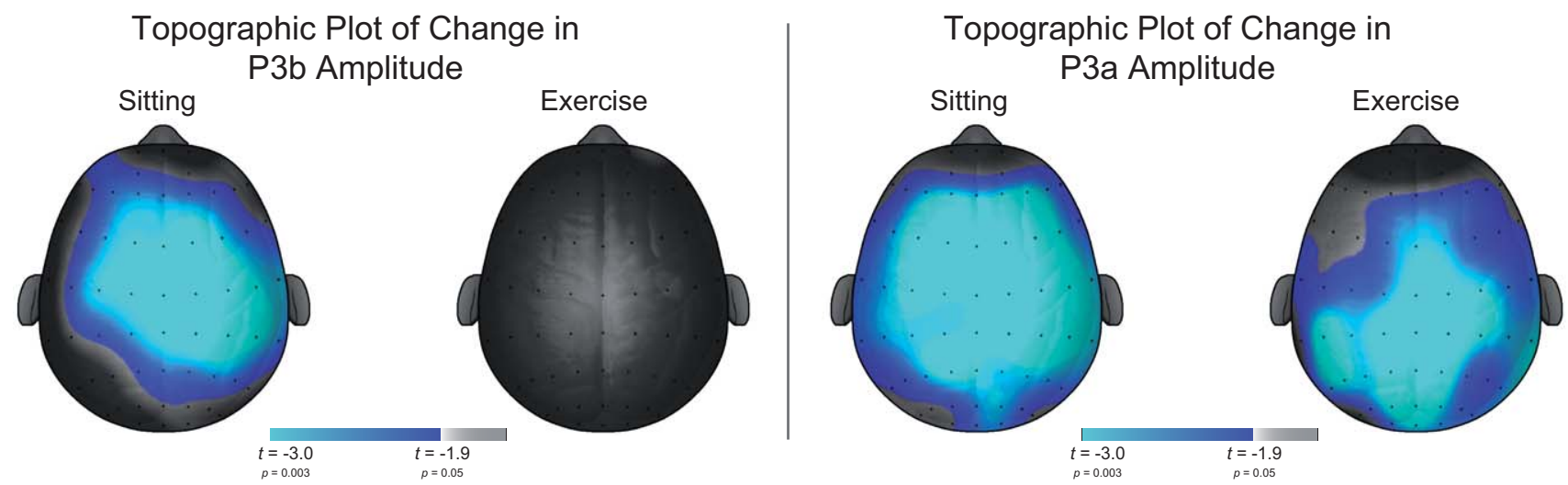

Figure 2. Topographic maps of the statistical change from pre- to posttest in the P3b and P3a components for each session.

exercise condition, $t(33)=0.2, p=.82, d=-0.78$ (see Figure 2 ). Examination of change in P3b Amplitude (posttest P3b amplitude minus pretest $\mathrm{P} 3 \mathrm{~b}$ amplitude) $\times$ Mode revealed a greater reduction in $\mathrm{P} 3 \mathrm{~b}$ amplitude following the sitting condition $(-1.7 \pm 3.2 \mu \mathrm{V})$ than was observed following the exercise condition $(0.1 \pm 2.4 \mu \mathrm{V})$, $t(33)=3.6, p=.001, d=-0.89$. Examining differences between mode at each time point revealed no differences in P3b amplitude between the sitting $(12.3 \pm 5.8 \mu \mathrm{V})$ and exercise $(11.9 \pm 6.9 \mu \mathrm{V})$ conditions at pretest, $t(33)=0.8, p=.39, d=0.17$. At posttest, however, smaller P3b amplitude was observed for the sitting $(10.6 \pm 5.3 \mu \mathrm{V})$ condition relative to the exercise $(12.0 \pm 6.2 \mu \mathrm{V})$ condition, $t(33)=2.0, p=.05, d=-0.52$. A main effect of site was also observed, $F(6,33)=52.6, p<.001, \eta^{2}=.62$, with larger P3b amplitude at $\mathrm{Pz}$ relative to the $\mathrm{Fz}, \mathrm{FCz}, \mathrm{Cz}, \mathrm{CPz}$, and $\mathrm{Oz}$ electrode sites, $t \mathrm{~s}(33) \geq 5.4, p \mathrm{~s}<.001$; at $\mathrm{POz}$ relative to the $\mathrm{Fz}, \mathrm{FCz}, \mathrm{Cz}$, and Oz electrode sites, $t \mathrm{~s}(33) \geq 4.2, p \mathrm{~s}<.001$; at $\mathrm{CPz}$ relative to the $\mathrm{Fz}$, $\mathrm{FCz}$, and $\mathrm{Cz}$ electrode sites, $t \mathrm{~s}(33) \geq 7.0, p s<.001$; at $\mathrm{Cz}$ relative to the $\mathrm{Fz}$ and $\mathrm{FCz}$ electrode site, $t \mathrm{~s}(33) \geq 8.3, p \mathrm{~s}<.001$; and at $\mathrm{FCz}$ and $\mathrm{Oz}$ relative to the Fz electrode site, $t \mathrm{~s}(33)>5.6, p \mathrm{~s}<.001$. No interactions with site were observed, $F \mathrm{~s}(6,28) \leq 1.9, p \mathrm{~s} \geq .14$, $\eta^{2} \mathrm{~s} \leq .05$.

Analysis of P3b latency revealed a main effect of time, $F(1,33)=7.0, p=.012, \eta^{2}=.18$, with longer P3b latency at posttest $(421.7 \pm 36.7 \mathrm{~ms})$ relative to pretest $(414.1 \pm 41.7 \mathrm{~ms})$. A main effect of site, $F(6,33)=10.1, p<.001, \eta^{2}=.23$, was observed with shorter $\mathrm{P} 3 \mathrm{~b}$ latency at $\mathrm{Oz}$ relative to the $\mathrm{Fz}, \mathrm{FCz}, \mathrm{Cz}$, and $\mathrm{CPz}$ electrode sites, $t \mathrm{~s}(33) \geq 3.3, p \mathrm{~s} \leq .002$; at $\mathrm{POz}$ relative to the $\mathrm{Fz}$, $\mathrm{FCz}$, and $\mathrm{Cz}$ electrode sites, $t \mathrm{~s}(33) \geq 3.3, p \mathrm{~s} \leq .002$; and at $\mathrm{Pz}$ relative to the $\mathrm{FCz}$ electrode site, $t(33)=3.5, p=.001$. No main effects of mode, $F(1,33)=0.6, p=.44, \eta^{2}=.02$, interactions of Mode $\times$ Time, $F(1,33)=0.7, p=.42, \eta^{2}=.02$, or interactions with site, $F \mathrm{~s}(6,28) \leq 0.5, p \mathrm{~s} \geq .69, \eta^{2} \mathrm{~s} \leq .02$, were observed. Examination of changes in P3b latency (posttest P3b latency minus pretest P3b latency) similarly revealed no main effects of mode or site, nor interactions of Mode $\times$ Site, $F \mathrm{~s}(1,28) \leq 0.7, p s \geq .42, \eta^{2} \mathrm{~s} \leq .12$.

\section{P3a ERP Component}

Analysis of P3a amplitude revealed a main effect of time, $F(1,33)=24.2, p<.001, \eta^{2}=.42$, with smaller P3a amplitude at posttest $(14.3 \pm 5.2 \mu \mathrm{V})$ relative to pretest $(16.4 \pm 3.8 \mu \mathrm{V})$. A main effect of site, $F(6,33)=18.4, p<.001, \eta^{2}=.36$, was also observed with larger $\mathrm{P3a}$ amplitude at $\mathrm{Cz}$ and $\mathrm{CPz}$ relative to the $\mathrm{Fz}, \mathrm{FCz}$, and $\mathrm{Oz}$ electrode sites, $t \mathrm{~s}(33) \geq 3.8, p \mathrm{~s} \leq .001$; at $\mathrm{Pz}$ and $\mathrm{POz}$ rela- tive to the $\mathrm{Fz}$ and $\mathrm{Oz}$ electrode sites, $t \mathrm{~s}(33) \geq 4.1, p \mathrm{~s}<.001$; and at $\mathrm{FCz}$ relative to the $\mathrm{Fz}$ electrode site, $t(33)=10.0, p<.001$. No main effects of mode, $F(1,33)=0.5, p=.47, \eta^{2}=.02$; interactions of Mode $\times$ Time, $F(1,33)=1.9, p=.18, \eta^{2}=.05$; nor interactions with site were observed, $F_{\mathrm{s}}(6,28) \leq 2.4, p \mathrm{~s} \geq .08, \eta^{2} \mathrm{~s} \leq .07$. Replicating the larger model, examination of changes in P3a amplitude (posttest $\mathrm{P} 3 \mathrm{a}$ amplitude minus pretest $\mathrm{P} 3 \mathrm{a}$ amplitude) revealed no main effects of mode nor interactions of Mode $\times$ Site, $F \mathrm{~s}(1,28) \leq 1.9, p \geq .18, \eta^{2} \leq .16$.

Examination of P3a latency revealed a main effect of site, $F(6,33)=7.0, p=.001, \eta^{2}=.18$, with shorter $\mathrm{P} 3$ a latency at $\mathrm{FCz}$, $\mathrm{Cz}, \mathrm{CPz}, \mathrm{Pz}$, and $\mathrm{POz}$ relative to the Fz electrode site, $t \mathrm{~s}(33) \geq 3.6$, $p s \leq 0.001$. No main effects of mode, $F(1,33)=0.1, p=.72$, $\eta^{2}=.004$; time, $F(1,33)=0.003, p=.96, \eta^{2}<.001$; interactions of Mode $\times$ Time, $F(1,33)=0.1, p=.83, \eta^{2}=.001$; nor interactions with site were observed, $F \mathrm{~s}(6,28) \leq 1.7, p \mathrm{~s} \geq .18, \eta^{2} \mathrm{~s} \leq .05$ (see Figure 2). Examination of changes in P3a latency (posttest P3a latency minus pretest $\mathrm{P} 3 \mathrm{a}$ latency) replicated these findings with no main effects of mode or site, nor interactions of Mode $\times$ Site, $F_{\mathrm{s}}(1,28) \leq 0.6, p \geq .73, \eta^{2} \leq .12$.

\section{Discussion}

The aim of the present investigation was to provide new insight into the extent to which single bouts of aerobic intensities of exercise may differentially relate to two aspects of attention: attentional orienting as indexed by the P3a ERP, and the allocation of attentional resources during stimulus engagement as indexed by the P3b ERP. In contrast to our a priori hypothesis, findings revealed that, following a single 20-min bout of moderate intensity aerobic exercise, ERP component amplitude was maintained from pretest only for the P3b elicited by the oddball target stimulus while decreased P3b amplitude was observed following a similar duration of seated rest. No exercise-related effects were observed for the P3a ERP component elicited by the distractor stimulus.

Highlighting the importance of utilizing a within-subjects repeated measures design, findings revealed shorter reaction time for the exercise condition at both pre- and posttest, relative to the sitting condition. Should this investigation have only assessed cognition after each experimental condition, a conclusion that exercise can shorten reaction time might mistakenly have been reached. Indeed, replicating previous research (Kamijo et al., 2004; Nakamura et al., 1999), greater attentional resource allocation during stimulus engagement (as indexed by larger P3b amplitude) 
was observed following exercise relative to following rest. However, contrary to prior interpretations of exercise-induced enhancements in attentional processing, when examined relative to pretest, single bouts of physical activity appear to sustain such attentional processes, whereas sitting resulted in impairments. Thus, at least for simple stimulus discrimination tasks, it is not that exercise enhances attention but that prolonged sitting is detrimental to the allocation of attentional resources. Interestingly, such findings match well with previous research in the educational psychology domain suggesting attentional waning in the classroom (Bunce, Flens, \& Neiles, 2010; Olmsted, 1999). Specifically, declines in on-task behavior indicative of attention have been characteristically observed following 10 to $20 \mathrm{~min}$ of attentional engagement in the classroom, providing impetus to utilize lecture strategies that build in breaks at these time points (Bunce et al., 2010; Olmsted, 1999). Within the present investigation, participants were asked to engage in a challenging stimulus discrimination task lasting approximately $15 \mathrm{~min}$, watch $20 \mathrm{~min}$ of an educational video, and then complete an additional $15 \mathrm{~min}$ of the stimulus discrimination task. Thus, while participants did change activities every 15 to $20 \mathrm{~min}$, sitting was associated with decrements in the allocation of attentional resources at posttest relative to pretest. Within this context, then, although aerobic intensities of exercise did not enhance the P3b ERP component, the observation that attentional resource allocation was sustained cannot be discounted. That is, such findings provide support for the utilization of activity breaks during the day where such single bouts of physical activity may sustain attentional processing. Although the present investigation did not include measures of information retention from the educational video that was watched during the sitting and exercise conditions, clearly this is an area that warrants further attention to better understand how exercise might influence memory acquisition in a classroom setting.

Examination of the P3a ERP component within the present investigation suggests that such single bouts of aerobic intensities of exercise selectively impact attentional processes. Indeed, no differences in $\mathrm{P} 3 \mathrm{a}$ amplitude between sitting and exercise were observed, suggesting that focal attention resulting from attentional orienting does not appear to be sensitive to the influences of single bouts of aerobic physical activity. Acute exercise-induced changes in cognition, therefore, do not appear to originate from an overall modulation of attention but instead are specific to aspects of attentional processing. Some caution is warranted with such statements as previous research has observed that the amplitude of the P3a ERP component habituates as the novelty of the stimulus decreases with repeated presentations (Yamaguchi \& Knight, 1991). Such habituation, however, occurs rapidly within the first several presentations of the novel stimuli, and a key component of the present three-stimulus oddball task is not the novelty of the task-irrelevant stimuli but instead the visually salient characteristics of the checkerboard pattern to elicit the generation of the P3a ERP component (Hagen et al., 2006; Yamaguchi \& Knight, 1991). Given neuropharmacological evidence differentiating the P3a and P3b components (Polich, 2007), the present findings that only the $\mathrm{P} 3 \mathrm{~b}$ was influenced by acute bouts of aerobic physical activity appear to suggest that the mechanisms underlying exercise-induced modulations in cognition may be due to the locus-coeruleusnorepinephrine system. According to a theoretical perspective put forth by Nieuwenhuis et al. (2005), the locus-coeruleusnorepinephrine system serves to modulate information processing through tonic and phasic activation of the locus coeruleus. Within this theoretical context, high levels of tonic activity and lower phasic responses in the locus coeruleus allow for increases in neuronal responsively unrelated to goal-oriented behavior on the present task. Indeed, attention-deficit hyperactivity disorder (ADHD) is believed to be associated with high baseline levels of tonic activity in the locus coeruleus, which when coupled with reduced phasic activity of the locus coeruleus manifests in the characteristic reduction in $\mathrm{P} 3 \mathrm{~b}$ amplitude frequently reported in this population (Nieuwenhuis et al., 2005). The present findings of reductions in attentional processing following the sitting condition are also consonant with prior research, which has observed reductions in the amplitude of the P3b ERP during periods of inactivity when there are higher levels of tonic activity and lower phasic responses in the locus coeruleus (Nieuwenhuis et al., 2005).

However, when moderate levels of tonic activity in the locus coeruleus are present, phasic activity and associated releases of norepinephrine act to increase and maintain the responsivity of the synaptic functions of cortical neurons (Nieuwenhuis et al., 2005). Such regulatory actions thus allow for enhancements in top-down modulation of activity within the prefrontal cortex (Nieuwenhuis et al., 2005). Indeed, in support of such an assertion that physical activity may modulate attentional processing by way of the locuscoeruleus-norepinephrine system, prior research in humans has indicated that moderate intensity aerobic exercise increases norepinephrine within the blood plasma and that the levels of norepinephrine continue to increase for a period of time following the cessation of exercise (Dimsdale, Hartley, Guiney, Ruskin, \& Greenblatt, 1984). Although much of these increases in norepinephrine within the blood plasma may be the result of sympathetic innervation of the heart and active skeletal muscles (Buckworth \& Dishman, 2002), evidence from nonhuman animal models also indicates that treadmill exercise appears to protect against depletion of norepinephrine within the locus coeruleus, amygdala, and hippocampus (Dishman, Renner, White-Welkley, Burke, \& Bunnell, 2000). Thus, it may be that these single bouts of physical activity act to regulate the locus-coeruleus-norepinephrine system to maintain moderate levels of tonic activity in the locus coeruleus and allow for optimal phasic responses and norepinephrine release, which in turn facilitates/maintains attentional processing. Accordingly, given the findings of the present investigation, further research is necessary to better understand how these acute exerciseinduced modulations in cognition may relate to changes in the locus-coeruleus-norepinephrine system.

Despite the methodological strength of the present investigation, there are a number of limitations that warrant further discussion. First and foremost is the lack of any observed behavioral differences resulting from a single bout of aerobic exercise. Although the P3b ERP component has generally been observed to be sensitive to such bouts of exercise across a number of prior investigations, behavioral findings have been less consistently observed within high-functioning college-aged adults such as those utilized in the present investigation (Hillman et al., 2012). Such behavioral modulations are also distinctly absent when the cognitive task may not sufficiently tax neural resources such as in the case of stimulus discrimination tasks; but are more readily apparent for task or task conditions that tap aspects of high-level cognition, such as cognitive control (Hillman et al., 2012). Indeed, the vast majority of research in this area has utilized tasks that tap an aspect of cognitive control known as inhibitory control, which relates to the ability to act on the basis of choice rather than impulse (Davidson, Amso, Anderson, \& Diamond, 2006). Thus, while the present investigation provides evidence suggesting that aerobic exercise sustains, rather than enhances, aspects of attentional pro- 
cessing in the service of stimulus discrimination, the extent to which these findings may manifest in response to tasks that are more cognitively demanding is as of yet unknown. Also of importance to note is the timing of the cognitive assessment following exercise. Within the present investigation, ERP indices of attention were assessed approximately $4.5 \mathrm{~min}$ following the exercise bout, which is consistent with prior investigations assessing neuroelectric indices of attention in response to simple stimulus discrimination tasks (Kamijo et al., 2004; Nakamura et al., 1999). However, much of the existent literature assessing aspects of inhibitory control have delayed neuroelectric testing until 16 to 48 min postexercise (Hillman et al., 2003; Pontifex et al., 2013). Accordingly, further research is necessary to determine the extent to which the cognitive load imposed by a given task may interact with the timing of the cognitive assessment following a bout of exercise.

Further research is also necessary to better understand the extent to which individual differences may moderate the effects of exercise on cognition. That is, although the present investigation utilized a high-functioning population of college-aged adults in a within-subjects counterbalanced design, there was still considerable variability in the effects of exercise relative to sitting at the individual participant level. Thus, it may be that other individual difference factors such as cardiorespiratory fitness may modulate the effect of a single bout of exercise. A number of previous investigations have suggesting that a dose-response curve may exist for the exercise-cognition relationship such that the greatest effects are observed following exercise at intensities between $65-85 \%$ of maximal heart rate (Hillman et al., 2012). Although the present investigation was designed to assess the effects of a mod- erate intensity bout of aerobic exercise within this targeted window, this intensity was based on age-predicted maximal heart rate. Thus, individual differences in cardiovascular fitness may have shifted individuals toward the tail ends of this target intensity window, potentially reducing the impact of exercise. Conversely, however, findings relative to the effects of acute bouts of exercise on affect argue that a more appropriate method of prescribing exercise intensity is to set it in relation to a physiological marker of metabolic processes such as the ventilatory threshold or lactate threshold, as $70 \%$ of maximum heart rate or aerobic fitness may reflect very different metabolic processes for an individual with higher aerobic fitness than lower aerobic fitness (Ekkekakis, Parfitt, \& Petruzzello, 2011). Thus, further research is necessary to better understand how such individual difference factors and exercise intensity may modulate the observed relationship between exercise/sitting and cognition.

Collectively, findings from the present investigation indicate that modulations in cognition resulting from a single bout of moderately intense aerobic exercise appear to be specific to attentional processing rather than a more generalized mechanism, as focal attention resulting from attentional orienting (as indexed by the P3a ERP component) does not appear to be sensitive to the influences of these single bouts of physical activity. Contrary to prior interpretations of exercise-induced enhancements in attentional processing, these findings indicate that single bouts of physical activity appear to sustain such attentional processes involved in context updating, whereas prolonged sitting results in impairments. Thus, these data speak to the utilization of physical activity breaks as a means of maintaining high levels of attentional engagement.

\section{References}

Buckworth, J., \& Dishman, R. K. (2002). Determinants of exercise and physical activity. In Exercise psychology (pp. 191-209). Champaign, IL: Human Kinetics.

Bunce, D., Flens, E., \& Neiles, K. (2010). How long can students pay attention in class? A study of student attention decline using clickers. Journal of Chemical Education, 87, 1438-1443. doi: 10.1021/ ed $100409 \mathrm{p}$

Chatrian, G. E., Lettich, E., \& Nelson, P. L. (1985). Ten percent electrode system for topographic studies of spontaneous and evoked EEG activity. American Journal of EEG Technology, 25, 83-92.

Compumedics Neuroscan. (2003). Offline analysis of acquired dataSCAN 4.3 (Vol. II). El Paso, TX.

Conroy, M. A., \& Polich, J. (2007). Normative variation of P3a and P3b from a large sample: Gender, topography, and response time. Journal of Psychophysiology, 21, 22-32. doi: 10.1027/0269-8803.21.1.22

Cooter, S., Holt, C., \& Lachmann, M. (Producers). (2011). Wonders of the universe [Motion picture]. United States: BBC America.

Davidson, M. C., Amso, D., Anderson, L. C., \& Diamond, A. (2006). Development of cognitive control and executive functions from 4 to 13 years: Evidence from manipulations of memory, inhibition, and task switching. Neuropsychologia, 44, 2037-2078. doi: 10.1016/j .neuropsychologia.2006.02.006

Dimsdale, J., Hartley, H., Guiney, T., Ruskin, J., \& Greenblatt, D. (1984). Postexercise peril: Plasma catecholamines and exercise. Journal of the American Medical Association, 251, 630-632. doi: 10.1001/ jama.1984.03340290044018

Dishman, R. K., Renner, K. J., White-Welkley, J. E., Burke, K. A., \& Bunnell, B. N. (2000). Treadmill exercise training augments brain norepinephrine response to familiar and novel stress. Brain Research Bulletin, 52, 337-342. doi: 10.1016/S0361-9230(00)00271-9

Donchin, E. (1981). Surprise! ... Surprise? Psychophysiology, 18, 493513. doi: 10.1111/j.1469-8986.1981.tb01815.x

Ekkekakis, P., Parfitt, G., \& Petruzzello, S. J. (2011). The pleasure and displeasure people feel when they exercise at different intensities:
Decennial update and progress towards a tripartite rationale for exercise intensity prescription. Sports Medicine, 41, 641-671. doi: 10.2165/ 11590680-000000000-00000

Faul, F., Erdfelder, E., Lang, A-G., \& Buchner, A. (2007). G*Power 3: A flexible statistical power analysis program for the social, behavioral, and biomedical sciences. Behavior Research Methods, 29, 175-191. doi: 10.3758/BF03193146

Hagen, G. F., Gatherwright, J. R., Lopez, B. A., \& Polich, J. (2006). P3a from visual stimuli: Task difficulty effects. International Journal of Psychophysiology, 59, 8-14. doi: 10.1016/j.ijpsycho.2005.08.003

Hillman, C. H., Kamijo, K., \& Pontifex, M. B. (2012). The relation of ERP indices of exercise to brain health and cognition. In H. Boecker, C. H. Hillman, L. Scheef, \& H. K. Struder (Eds.), Functional neuroimaging in exercise and sport sciences, (pp. 419-446). New York, NY: Springer. doi: 10.1007/978-1-4614-3293-7_18

Hillman, C. H., Pontifex, M. B., Castelli, D. M., Khan, N. A., Raine, L. B., Scudder, M. R., ... Kamijo, K. (2014). Effects of the FITKids randomized controlled trial on executive control and brain function. Pediatrics, 134, e1063-e1071. doi: 10.1542/peds.2013-3219

Hillman, C. H., Pontifex, M. B., Raine, L. B., Castelli, D. M., Hall, E. E., \& Kramer, A. F. (2009). The effect of acute treadmill walking on cognitive control and academic achievement in preadolescent children. Neuroscience, 159, 1044-1054. doi: 10.1016/j.neuroscience.2009 .01 .057

Hillman, C. H., Snook, E. M., \& Jerome, G. J. (2003). Acute cardiovascular exercise and executive control function. International Journal of Psychophysiology, 48, 307-314. doi: 10.1016/S0167-8760(03)00080-1

Ilan, A. B., \& Polich, J. (1999). P300 and response time from a manual Stroop task. Clinical Neurophysiology, 110, 367-373. doi: 10.1016/ S0168-5597(98)00053-7

Kamijo, K., Hayashi, Y., Sakai, T., Yahiro, T., Tanaka, K., \& Nishihira, Y. (2009). Acute effects of aerobic exercise on cognitive function in older adults. Journal of Gerontology, Series B: Psychological Sciences, 64, 356-363. doi: 10.1093/geronb/gbp030 
Kamijo, K., Nishihira, Y., Hatta, A., Kaneda, T., Wasaka, T., Kida, T., \& Kuroiwa, K. (2004). Differential influences of exercise intensity on information processing in the central nervous system. European Journal of Applied Physiology, 92, 305-311. doi: 10.1007/s00421-004-1097-2

Knight, R. (1984). Decreased response to novel stimuli after prefrontal lesions in man. Electroencephalography and Clinical Neurophysiology, 59, 9-20. doi: 10.1016/0168-5597(84)90016-9

Kok, A. (2001). On the utility of P3 amplitude as a measure of processing capacity. Psychophysiology, 38, 557-577. doi: 10.1017/ S0048577201990559

Kumar, N., Singh, M., Sakshi, B., Roy, P. S., \& Behera, J. K. (2012). Effect of acute moderate exercise on cognitive P300 in persons having sedentary lifestyles. International Journal of Applied and Basic Medical Research, 2, 67-69. doi: 10.4103/2229-516X.96813

Lambourne, K., \& Tomporowski, P. (2010). The effect of exercise-induced arousal on cognitive task performance: A meta-regression analysis. Brain Research, 13, 12-24. doi: 10.1016/j.brainres.2010.03.091

Magliero, A., Bashore, T. R., Coles, M. G., \& Donchin, E. (1984). On the dependence of $\mathrm{P} 300$ latency on stimulus evaluation processes. Psychophysiology, 21, 171-186. doi: 10.1111/j.1469-8986.1984 .tb00201.x

Magnié, M., Bermon, S., Martin, F., Madany-Lounis, M., Suisse, G., Muhammad, W., \& Dolisi, C. (2000). P300, N400, aerobic fitness, and maximal aerobic exercise. Psychophysiology, 37, 369-377. doi: 10.1111/1469-8986.3730369

Nakamura, Y., Nishimoto, K., Akamatu, M., Takahashi, M., \& Maruyama, A. (1999). The effect of jogging on P300 event related potentials. Electromyography and Clinical Neurophysiology, 39, 71-74.

Nieuwenhuis, S., Aston-Jones, G., \& Cohen, J. D. (2005). Decision making, the P3, and the locus coeruleus-norepinephrine system. Psychological Bulletin, 131, 510-532. doi: 10.1037/00332909.131.4.510

Oldfield, R. C. (1971). The assessment and analysis of handedness: The Edinburgh inventory. Neuropsychologia, 9, 97-113. doi: 10.1016/00283932(71)90067-4

Olmsted, J. A. (1999). The mid-lecture break: When less is more. Journal of Chemical Education, 76, 525-527. doi: 10.1021/ed076p525

Peirce, J. W. (2009). Generating stimuli for neuroscience using PsychoPy. Frontiers in Neuroinformatics, 2, 10. doi: 10.3389/neuro.11.010.2008

Polich, J. (2007). Updating P300: An integrative theory of P3a and P3b. Clinical Neurophysiology, 118, 2128-2148. doi: 10.1016/j.clinph .2007.04.019

Polich, J., \& Criado, J. R. (2006). Neuropsychology and neuropharmacology of P3a and P3b. International Journal of Psychophysiology, 60, 172-185. doi: 10.1016/j.ijpsycho.2005.12.012
Polich, J., \& Kok, A. (1995). Cognitive and biological determinants of P300: An integrative review. Biological Psychology, 41, 103-146. doi: 10.1016/0301-0511(95)05130-9

Pontifex, M. B., Hillman, C. H., \& Polich, J. (2009). Age, physical fitness, and attention: P3a and P3b. Psychophysiology, 46, 379-387. doi: 10.1111/j.1469-8986.2008.00782.x

Pontifex, M. B., Saliba, B. J., Raine, L. B., Picchietti, D. L., \& Hillman, C. H. (2013). Exercise improves behavioral, neurocognitive, and scholastic performance in children with ADHD. Journal of Pediatrics, 162, 543551. doi: 10.1016/j.jpeds.2012.08.036

Rushby, J. A., Barry, R. J., \& Doherty, R. J. (2005). Separation of the components of the late positive complex in an ERP dishabituation paradigm. Clinical Neurophysiology, 116, 2363-2380. doi: 10.1016/ j.clinph.2005.06.008

Scudder, M. R., Drollette, E. S., Pontifex, M. B., \& Hillman, C. H. (2012). Neuroelectric indices of goal maintenance following a single bout of physical activity. Biological Psychology, 89, 528-531. doi: 10.1016/ j.biopsycho.2011.12.009

Squires, N. K., Squires, K. C., \& Hillyard, S. A. (1975). Two varieties of long-latency positive waves evoked by unpredictable auditory stimuli in man. Electroencephalography and Clinical Neurophysiology, 38, 387401. doi: 10.1016/0013-4694(75)90263-1

Thomas, S., Reading, J., \& Shephard, R. J. (1992). Revision of the physical activity readiness questionnaire (PAR-Q). Canadian Journal of Sport Sciences, 17, 338-345.

Verburgh, L., Königs, M., Scherder, E. J., \& Oosterlaan, J. (2014). Physical exercise and executive functions in preadolescent children, adolescents and young adults: A meta-analysis. British Journal of Sports Medicine, 48, 973-979. doi: 10.1136/bjsports-2012-091441

Walhovd, K. B., Rosquist, H., \& Fjell, A. M. (2008). P300 amplitude age reductions are not caused by latency jitter. Psychophysiology, 45, 545553. doi: 10.1111/j.1469-8986.2008.00661.x

Yagi, Y., Coburn, K. L., Estes, K. M., \& Arruda, J. E. (1999). Effects of aerobic exercise and gender on visual and auditory P300, reaction time, and accuracy. European Journal of Applied Physiology, 80, 402-408. doi: 10.1007/s004210050611

Yamaguchi, S., \& Knight, R. T. (1991). Age effects on the P300 to novel somatosensory stimuli. Electroencephalography and Clinical Neurophysiology, 78, 297-301. doi: 10.1016/0013-4694(91)90184-6

(Received August 15, 2014; ACCEPTED November 15, 2014) 